\title{
Brain Stroke Detection by Microwaves Using Prior Information from Clinical Databases
}

\author{
Natalia Irishina ${ }^{1}$ and Aurora Torrente ${ }^{2}$ \\ ${ }^{1}$ Instituto Gregorio Millán, Universidad Carlos III de Madrid, 28911 Leganés, Madrid, Spain \\ ${ }^{2}$ Departamento de Ciencia e Ingeniería de Materiales e Ingeniería Química, Universidad Carlos III de Madrid, \\ 28911 Leganés, Madrid, Spain
}

Correspondence should be addressed to Natalia Irishina; nirishin@math.uc3m.es

Received 18 December 2012; Revised 22 April 2013; Accepted 24 April 2013

Academic Editor: Bashir Ahmad

Copyright (C) 2013 N. Irishina and A. Torrente. This is an open access article distributed under the Creative Commons Attribution License, which permits unrestricted use, distribution, and reproduction in any medium, provided the original work is properly cited.

\begin{abstract}
Microwave tomographic imaging is an inexpensive, noninvasive modality of media dielectric properties reconstruction which can be utilized as a screening method in clinical applications such as breast cancer and brain stroke detection. For breast cancer detection, the iterative algorithm of structural inversion with level sets provides well-defined boundaries and incorporates an intrinsic regularization, which permits to discover small lesions. However, in case of brain lesion, the inverse problem is much more difficult due to the skull, which causes low microwave penetration and highly noisy data. In addition, cerebral liquid has dielectric properties similar to those of blood, which makes the inversion more complicated. Nevertheless, the contrast in the conductivity and permittivity values in this situation is significant due to blood high dielectric values compared to those of surrounding grey and white matter tissues. We show that using brain MRI images as prior information about brain's configuration, along with known brain dielectric properties, and the intrinsic regularization by structural inversion, allows successful and rapid stroke detection even in difficult cases. The method has been applied to $2 \mathrm{D}$ slices created from a database of $3 \mathrm{D}$ real MRI phantom images to effectively detect lesions larger than $2.5 \times 10^{-2} \mathrm{~m}$ diameter.
\end{abstract}

\section{Introduction}

A stroke or "brain attack" occurs when the blood flow to an area of the brain is interrupted by a blocked (ischemic stroke) or burst (hemorrhagic stroke) blood vessel. As a consequence, brain cells begin to die, and the abilities controlled by that part of the brain are lost. Stroke is a major cause of adult disability and death in the world, and the appropriate response to brain attack is emergency action: every minute people lose getting treatment increases the chances of them experiencing strokerelated disabilities or death. The symptoms of a stroke are varied and include weakness, clumsiness, altered feeling on one side of the body, speech disturbance, loss of vision, or dizziness [1]. Clinical diagnosis typically relies on medical imaging methods such as computed tomography (CT) and magnetic resonance imaging (MRI). Although these techniques are effective for identifying the location and type of a stroke, they are not suitable for being used in emergencies because of their high cost and lack of rapidity and portability.
Recently, the authors in [2] proposed a method based on microwave tomography (MWT), to supplement these medical imaging techniques, and focused on stroke detection. The authors used the nonlinear Newton reconstruction to obtain biologically meaningful images of the brain, including images of strokes. One of their important conclusions is that a multifrequency approach significantly improves the detection. Since it has been shown that the tissue malignancies, blood supply, hypoxia, acute ischemia, and chronic infarction present dielectric contrast compared with healthy tissues [3-6], MWT is a potentially good alternative to the medical imaging methods mentioned above. However, the results reported by [2] only provide an initial guidance to stroke diagnosis, which does not take into account dispersion of tissue dielectric properties or any particulars of head geometry.

Later, the authors in $[7,8]$ presented their simulation results of wave propagation in an anatomic realistic head phantom to detect brain matter affected by a simulated 
stroke, using a finite-difference time domain method (FDTD) to calculate the electromagnetic fields. Nevertheless, the detection of strokes in those articles is restricted to large dimension lesions situated close to the skull and does not provide accurate information about stroke location or size, their location errors varying from $5.68 \times 10^{-3} \mathrm{~m}$ to $4 \times 10^{-2} \mathrm{~m}$.

In this paper, we propose a method to detect strokes based on the propagation of microwaves in brain tissues. As in [8], our approach makes use of a (numerical) finitedifference method, but in the frequency-domain (FDFD), to estimate the electromagnetic fields as predicted by Maxwell's equations, supplied by the Sommerfeld boundary condition, and solves the associated inverse problem, which consists in minimizing the mismatch between the signal of the scattered microwaves and the one simulated with a numerical model. This technique has been successfully applied in the early detection of breast cancer [9]; however, the breast does not present the difficulty found when exposing the brain to microwaves, as it is surrounded by a skin layer, whereas the skull represents a dielectric shield within which the brain is confined. Moreover, the interior cephalic liquid has dielectric properties similar to those of blood, which reduces the contrast. Thus, high microwave frequencies, which lead to better resolution, are not able to penetrate into the brain, and consequently lower frequencies should be used instead, which leads to a loss of sensitivity to dielectric changes. To reconstruct the brain image, we take advantage of a multifrequency approach [2] and two well-known strategies that act like regularization methods: (1) the use of prior information, namely, known dielectric properties of brain tissues [10] and brain structure data, obtained from an MRI database [11], and (2) the level set technique for shape reconstruction [9]. On the other hand, detection in $2 \mathrm{D}$ is more computationally efficient than 3D models and allows rapidly detecting a potential stroke by varying the height at which microwave measurements are carried out.

Our study numerically demonstrates the potential use of microwaves for rapid discovery of brain lesions of sizes larger than $2.5 \times 10^{-2} \mathrm{~m}$, using a realistic model derived from real MRI images, despite the low penetration of microwaves into the brain. The use of the constant average map as initial guess leads to unsatisfactory stroke detection, and to overcome this difficulty, it is essential to obtain a good initial guess. Thus, we make use of an existing database of MRI images from normal brains and introduce two methods to build appropriate initial guesses; the first one is based on computing an "average brain configuration map" from the MRI database, and the second one is derived from the statistics of this set of images. In our experiments, once the initial guess is chosen, the next step consists in introducing and evolving the level set function, which estimates the location of potential lesions. The combination of both regularization techniques provides an imaging of the brain in which stroke location is well estimated in a high percentage of cases, even for strokes of diameter $3 \times 10^{-2} \mathrm{~m}$ profoundly situated in the brain.

Additionally, to compare these methods to traditional techniques, we also consider the average dielectric constant map to which we apply the classical pixel by pixel adjoint scheme, as in [9], to obtain an alternative initial guess; however, our techniques are faster and provide better quality brain images, which facilitate clinical diagnosis.

This paper is organized as follows. In Section 2, we describe the theoretical basement of the direct problem related with stroke detection and the brain models used in this study; in Section 3, we underline the measurement details, the choices of the initial guesses, and the results of the numerical experiments; finally, in Section 4, we summarize the main results of our study.

\section{Methods}

2.1. Direct Problem. Many interesting physical phenomena in electromagnetism can be described by a scalar approximation of Maxwell's equations. In our application, for example, rather than solving the three dimensional problems for brain, which is computationally expensive, we use a $2 \mathrm{D}$ domain at different brain axial cross-sections when searching for a stroke. The measurement is carried out by 50 equidistant receivers and transmitters set around the phantom head, which, respectively, measure and create electromagnetic fields. In the case described above, the scalar Helmholtz equation in the domain $\Omega$

$$
\Delta u(\mathbf{x})+\kappa(\mathbf{x}) u(\mathbf{x})=-q(\mathbf{x})
$$

is a good approximation for describing the nonzero component of the electric field $u$. To solve it, we use the standard Sommerfeld radiation condition in two dimensions at infinity

$$
\lim _{|\mathbf{x}| \rightarrow \infty} \sqrt{|\mathbf{x}|}\left(\frac{\partial u}{\partial|\mathbf{x}|}-i \kappa u\right)=0
$$

as boundary condition. In (1), $q(\mathbf{x})$ is the active source, and $\kappa(\mathbf{x})$ is the squared complex wavenumber, which is related to the complex relative permittivity through formula $\kappa=$ $\omega^{2} \mu_{0} \epsilon_{0} \epsilon_{r}^{*}$. Here, $\omega$ is the angular frequency of the testing waves, and $\mu_{0}$ and $\epsilon_{0}$ are the vacuum permeability and permittivity, respectively. Since we use a Sommerfeld radiation condition as a boundary condition, the computational domain $\Omega$ is discretized including appropriate boundary layers (PMLs) which absorb the radiation providing that no waves are coming from outside. With the Sommerfeld radiation condition, the Helmholtz equation has a unique solution.

Since we assume that the stroke area and the surrounding tissues are separated by sharp interfaces, we introduce the level set function $\phi(\mathbf{x})$ to characterize the location and shape of possible lesions. The inclusion of the shape description based on the level set function acts as a potential regularizing factor, stabilizing the inverse reconstruction. Our goal is to minimize iteratively the residual operator $R(\kappa)$, which describes the mismatch between the physically measured data and those corresponding to the guessed distribution of $\kappa$ in the domain of interest.

We start with $\phi(\mathbf{x})$ constant in all the domains. To evolve it, we define the forcing term $f(\mathbf{x})$ by

$$
f^{(n)}(\mathbf{x}, t)=-C\left[\operatorname{Re}\left(R^{\prime}(\kappa)^{*} R(\kappa)\right) \frac{\partial \kappa}{\partial \phi}\right] .
$$


Here, $R^{\prime}(\kappa)^{*} R(\kappa)$ is the Fréchet derivative of the residual operator $R(\kappa)$ with respect to $\kappa$, and the forcing term points in the descent direction of the least squares cost; $t$ is the artificial iteration time, and $C$ is a conveniently chosen constant. The Fréchet derivative can be calculated efficiently using the adjoint formulation (see $[9,12])$. Numerically discretizing, we arrive at the following iteration rule for level function updates:

$$
\phi^{(n+1)}=\phi^{(n)}+\delta t f^{(n)} .
$$

2.2. Head Model. It is well known that the interaction between biological tissues and electromagnetic radiation is strongly influenced by the variation in water content (e.g., blood contains mostly water molecules). The complex permittivity $\epsilon_{r}^{*}$ of a dielectric medium is defined as

$$
\epsilon_{r}^{*}=\epsilon_{r}+i \frac{\sigma}{\omega \epsilon_{0}}
$$

where $\epsilon_{r}$ denotes the relative permittivity, $\epsilon_{0}$ the vacuum permittivity, $\sigma$ the conductivity, and the symbol $i$ the imaginary unit. The efficient microwave frequencies, that is, those for which microwaves can penetrate inside the brain through the skull, are in the range of $(0.5-1) \times 10^{9} \mathrm{~Hz}$ [8]. The values of the relative permittivity and conductivity of brain tissues for this range of frequencies are well known $[10,13]$ and can be efficiently used as prior information. The maximal permittivity and conductivity values correspond to cerebral spin fluid $\left(\epsilon_{r}=68, \sigma=2.46\right)$ and blood $\left(\epsilon_{r}=61, \sigma=1.58\right)$, while the minimal values are for skull and fat inclusions $\left(\epsilon_{r}=\right.$ $12, \sigma=0.16$ and $\epsilon_{r}=5, \sigma=0.05$, correspondingly).

A second source of prior information consists in statistical summary brain configurations, extracted from an arbitrary normal brain image database. In the preparation of this paper, we obtained a collection of 100 three-dimensional MRI scans, corresponding to normal (control) patients from the Parkinson's Progression Markers Initiative (PPMI) database (http://www.ppmi-info.org/data), at the Image Data Archive at the Laboratory of Neuro Imaging (IDA) [11]. Each 3D image comprised around 250 two-dimensional slices corresponding to views of the brain acquired in the sagittal plane, at different positions. We used those $2 \mathrm{D}$ images to obtain realistic numerical phantoms and also to obtain data for simulated brain stroke patients. Figure 1 illustrates the three-dimensional configuration of these MRI brain images, visualized by means of the noncommercial medical visualization software Mango (http://ric.uthscsa.edu/mango/).

After collecting the MRI images of 100 control patients, at similar heights, we labelled them with indices $i=1, \ldots, 100$ and assigned dielectric properties to the axial cross-sections by mapping the real MRI image intensities to known dielectric parameter ranges [10]. Finally, hemorrhagic strokes were simulated by including ellipses of different sizes. The stroke dielectric properties correspondent to blood lesions were taken from $[10,13]$.

\section{Numerical Experiments}

3.1. Measurement Configuration. In our experiments, the imaging domain is $0.26 \times 0.26 \mathrm{~m}^{2}$. The antennas are situated equidistantly on a circle of $0.11 \mathrm{~m}$ radius, emitting microwaves of frequencies $(0.3-1) \times 10^{9} \mathrm{~Hz}$ by steps of $0.1 \times$ $10^{9} \mathrm{~Hz}$, and receiving the scattered signal. Since the shape of the skull can be averaged for patients of similar age and approximated using the structural inversion algorithm from the scattered waves [9], we can assume at this stage of the study that the configuration of the patient skull is known. The surrounding media are supposed to be air atmosphere. For the reconstruction, we use a mesh of $160 \times 160$ pixels, where each pixel is a square of $(1.625 \times 1.625) \times 10^{-6} \mathrm{~m}^{2}$. A Gaussian noise of $5 \%$ is added to the numerically simulated "true" data.

3.2. Brain Initial Guesses. Given the difficulty of the inverse problem for brain reconstruction because of ill-posedness, the use of the appropriate initial guess is decisive. We have used three different initial guesses. Firstly, we consider the ADC map, which is commonly used [9], applying afterwards the classical pixel by pixel reconstruction (PPS).

Secondly, we have derived two new initial guesses from a collection of 100 real clinical MRI images from control patients. It is logical to suppose that brain tissues of different patients have generally similar dielectric properties. Nevertheless, this internal brain tissue distribution cannot be recovered from microwave data because of low penetration. Thus, to obtain the second initial guess, we propose the average map method (AMM), consisting in computing an "average brain configuration" deduced from the average distribution of MRI intensities and, consequently, tissues densities, which are directly related to the average distributions of dielectric properties. To calculate the AMM initial guess for a given patient, the MRI skull dimensions are resized to the size of our patient's skull.

The other new technique is the representative method $(\mathrm{RM})$, where the initial guess is obtained as follows. Given the $n$ permittivity maps of the reference brains, which are resized as in the AMM method, all the pairwise differences are calculated ( $n=100$ in our experiments). Consequently, the maximal singular values of such difference maps are found using the matrix spectral norm, $\|\cdot\|_{2}$, which provides a vector $D_{i}$ of length $n-1$ for each of the $n$ brains in our prior data set. The mean value $\bar{D}_{i}, i=1,2, \ldots, n$, of the components of such vectors can be seen as an average distance between the $i$ th brain configuration and the rest of brain maps. Figure 2(a) depicts the distance distributions of the 10 brains with the lowest values of $\bar{D}_{i}$. Moreover, since they have different dispersions, we calculated the variances of $D_{i}, \sigma_{i}^{2}$, for all brains; the distributions of those with the 10 lowest values of $\sigma_{i}^{2}$ are shown in Figure 2(b). Finally, the brain chosen as the one that best represents the collection of $n$ brain maps (the most representative map) is the one with the smallest $\bar{D}_{i}$, provided that the corresponding dispersion is also small. In our experiments, the brain map with label $i=86$ has both one of the smallest means and variances, and therefore this is the one used as the third initial guess. 


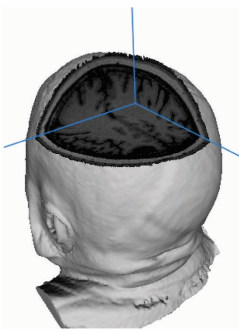

(a)

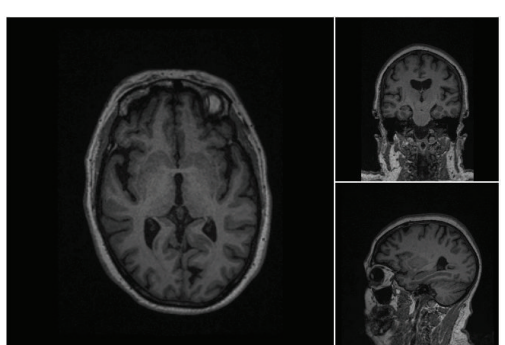

(b)

FIGURE 1: (a) Three-dimensional configuration of brain MRI images from the database used to obtain prior information for the proposed initial guesses. The head surface is rendered using the medical visualization software Mango. (b) Axial (left), coronal (upper right), and sagittal (lower right) views of the brain.

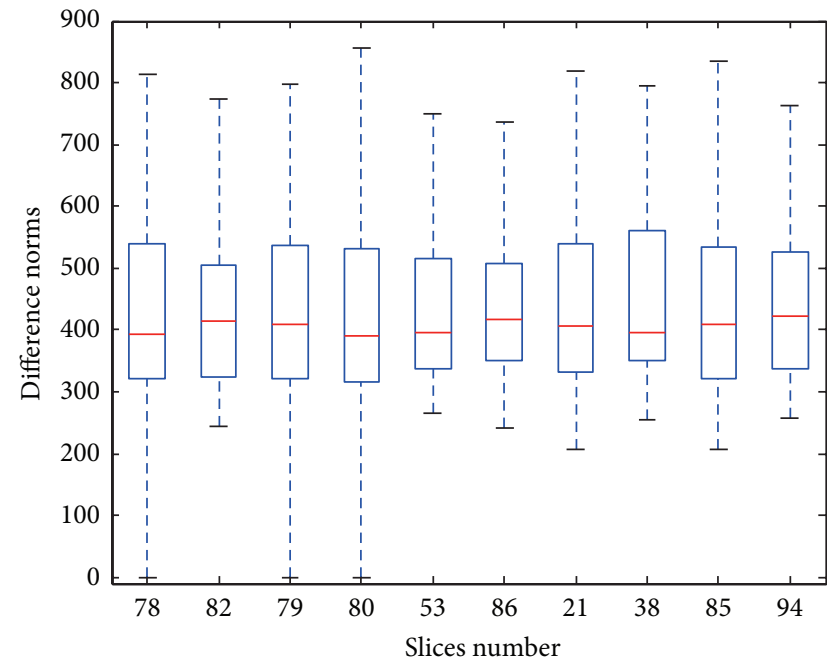

(a)

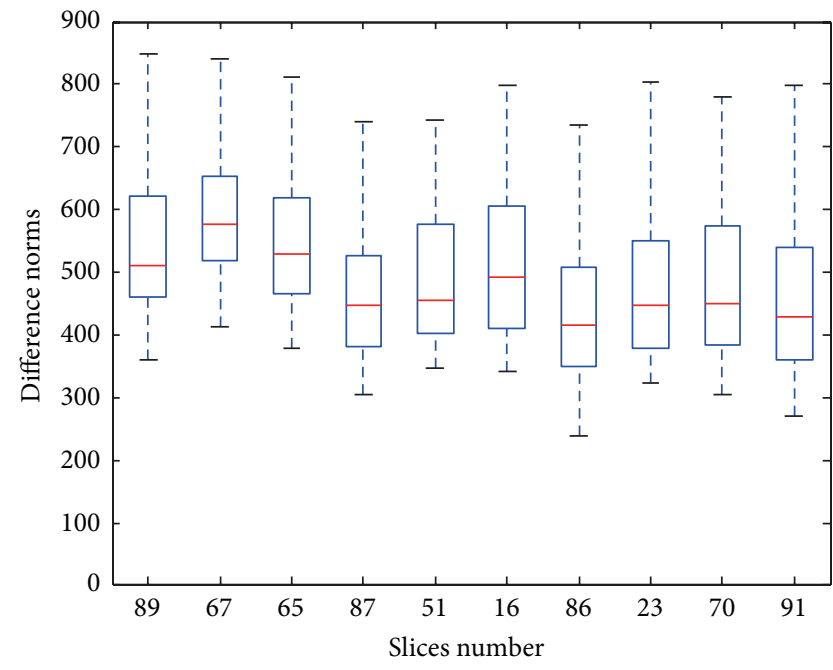

(b)

FIGURE 2: (a) Singular value distributions of the pairwise differences of the permittivity maps, $D_{i}$, corresponding to the 10 maps with the smallest mean value, $\bar{D}_{i}$. (b) Singular value distributions of the pairwise differences of the permittivity maps, $D_{i}$, corresponding to the 10 maps with the smallest variance, $\sigma_{i}^{2}$.

3.3. Stroke Location Results. After one of the initial guesses described above is selected, the location of the stroke is obtained by introducing the level set function with initial value equal to a positive constant in all the domains, where the domain is represented by either: the ADC, followed by pixel by pixel reconstruction (we name this the ADC-PPS map), the AMM map, or the RM map. The level set function is evolved using (3) and (4), implicitly representing the shape of the brain lesion. In our experiments, different locations and sizes of brain strokes have been analysed for detection. For example, the AMM algorithm has a probability of 0.96 of detecting profound strokes (for 96 out of 100 patients with a lesion of size $3 \times 10^{-2} \mathrm{~m}$ the detection was successful), whereas for the RM technique, this probability is 0.82 .

The results of brain lesion detection for the same patient with different stroke positions are shown in Figure 3. Each row represents a different method of detection, depending on which initial guess is used: the first one shows the ADCPPS case, the second one the RM case, and the third one the AMM case. The largest diameter of the stroke, which is situated deeply inside the brain, varies from $2.5 \times 10^{-2} \mathrm{~m}$ to $4.5 \times 10^{-2} \mathrm{~m}$. The left column in Figure 3 plots the patient's "real" brain configuration, and the central column shows the results of the reconstruction. Finally, the right column illustrates the evolution of the residuals in the algorithm. In the first case (ADC-PPS), this evolution presents a small jump when the level set function is introduced after the PPS stage; note that with this method, the detection process needs more iterations compared to the other two. In all the experiments, the stroke is successfully detected and located. Small areas of cephalic liquid are detected as well, but they can be ruled out as "stroke candidate" since their location is characteristically close to the skull.

To assess the effectiveness of the fastest methods, that is, with the AMM and RM initial guesses, Figure 4 shows the results of the detection for different patients and positions of the lesion. The rows are ordered from upper to lower according to decreasing sizes of the simulated stroke, with 

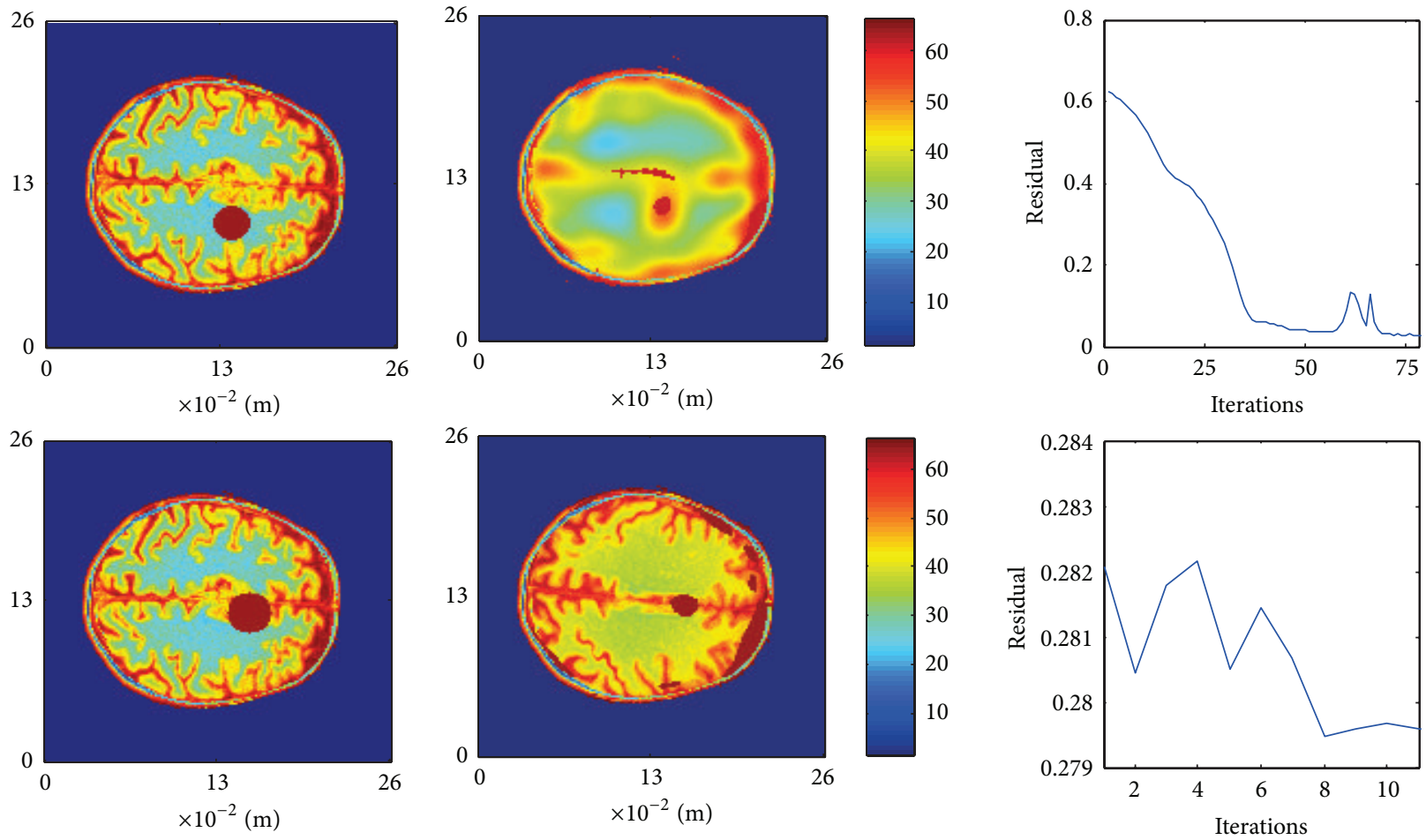

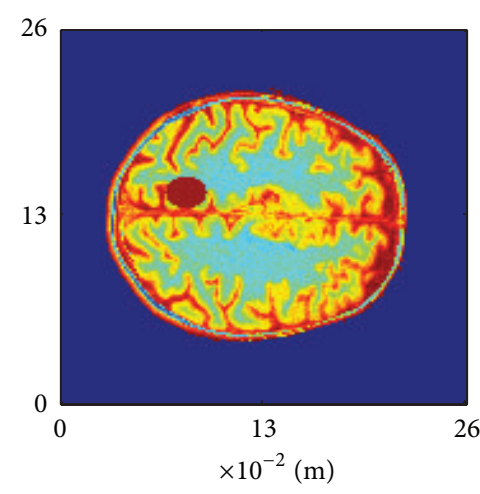

(a)

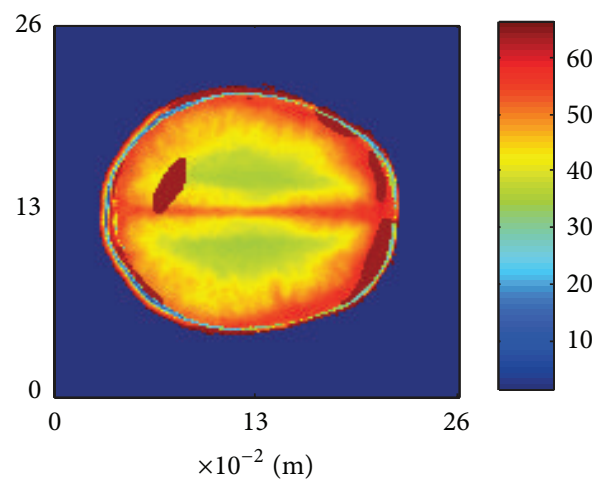

(b)

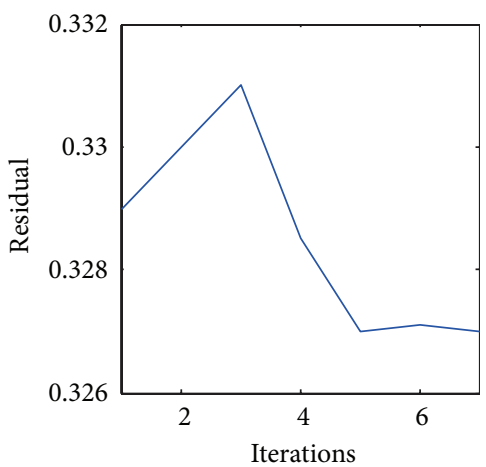

(c)

FIGURE 3: Comparison of the results obtained with the proposed initial guesses. The left column is the patient's brain configuration, the central column depicts the results of reconstruction, and the right column shows the residuals evolution. The rows correspond, from upper to lower, to the ADC-PPS, RM, and AMM initial guesses.

the largest diameter of the ellipse varying from $6.27 \times 10^{-2} \mathrm{~m}$ to $2.5 \times 10^{-2} \mathrm{~m}$. In particular, the first row corresponds to the detection of a stroke as in [8]. Again, the first column represents the patient's brain configuration, and the second one shows the RM reconstruction results, while the third one corresponds to the AMM results. The detection and location have been successful and precise in all the cases, even for profoundly situated strokes.

Finally, in Figure 5, we show that in case of healthy brain, the resulting maps do not present areas of abnormality. The left panel corresponds to the patient's brain map, the middle panel is the result of the reconstruction using the RM initial guess, and the right panel displays the results with the AMM initial guess. Few "ghosts" appear in the RM case, which can be ruled out because of clear symmetry.
As mentioned before, the main advantage of using the AMM and RM initial guesses, which are obtained from the MRI database, is that the detection is rapid compared to the ADC-PPS case. Using a standard PC $(2.66 \mathrm{GHz}$ and 3.25 GB RAM), the detection for those initial guesses takes only a few minutes, whereas in the ADC-PPS case, the number of iterations is roughly ten times larger, which for a multifrequency approach turns out to be computationally expensive, specially in the case of stroke detection, where a rapid diagnosis is crucial. In Table 1, we show the time (in seconds) needed for stroke detection; in all the cases, the stopping criterium is that the variance of the residuals of the last three iterations is within given limit values.

An additional benefit of the AMM and RM techniques is that the brain map they provide is more realistic than 

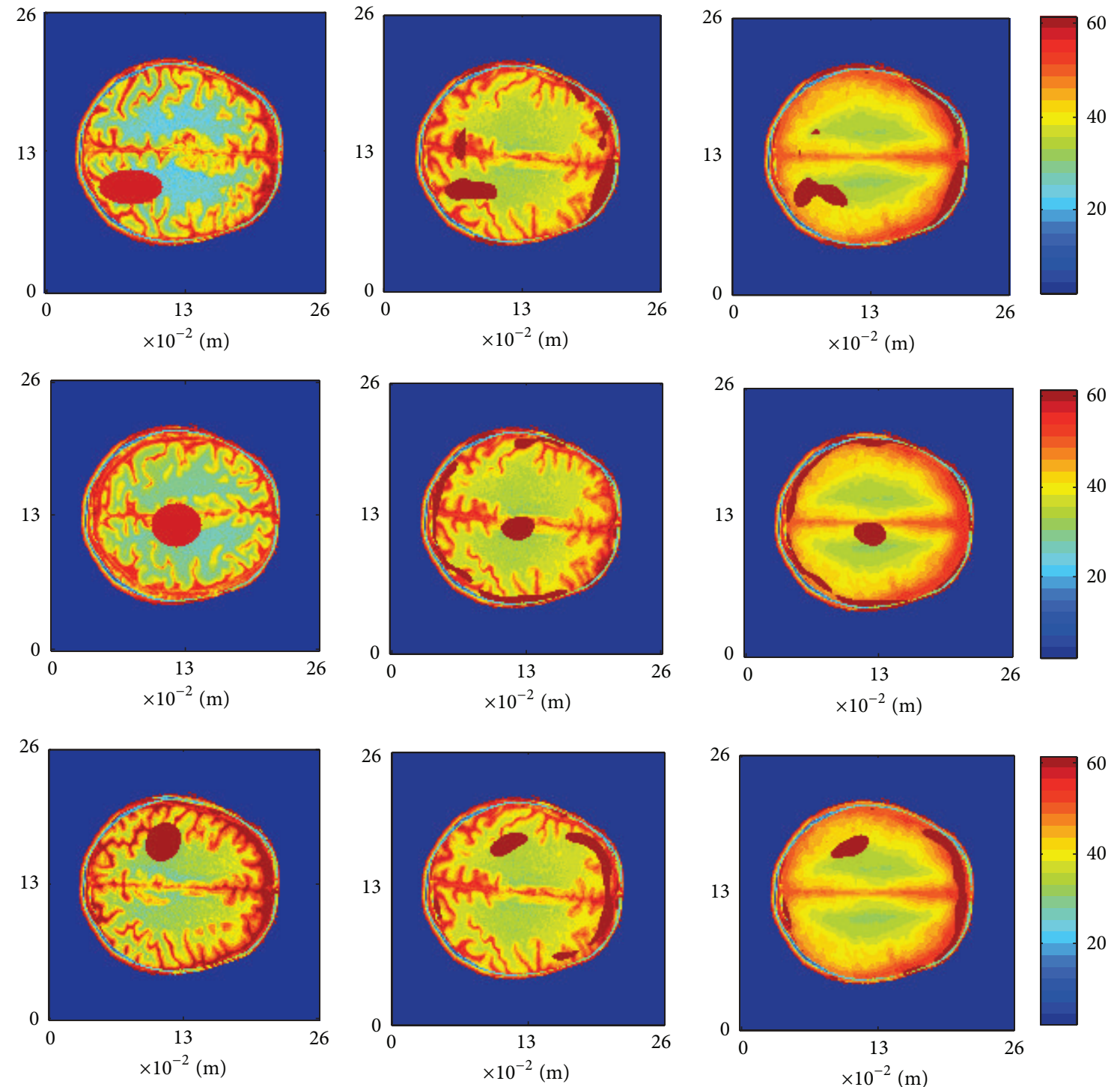

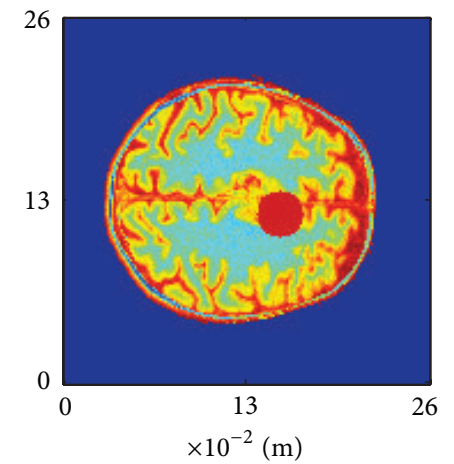

(a)

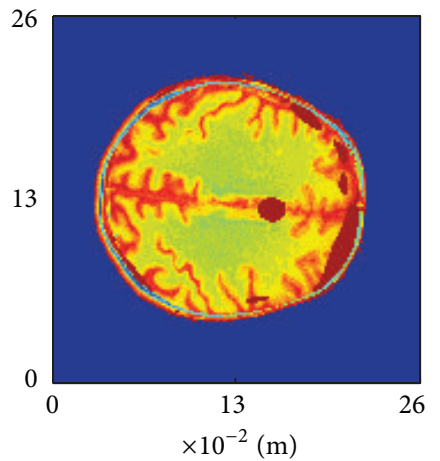

(b)

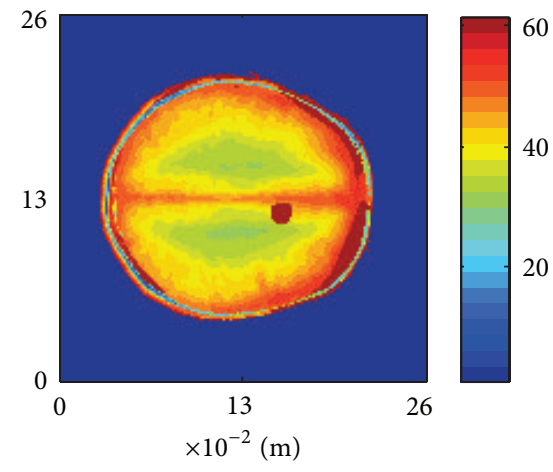

(c)

FIGURE 4: Reconstruction for different stroke locations and sizes, using RM (central column) and AMM (right column) initial guesses. The left column shows the patient's brain configuration. 


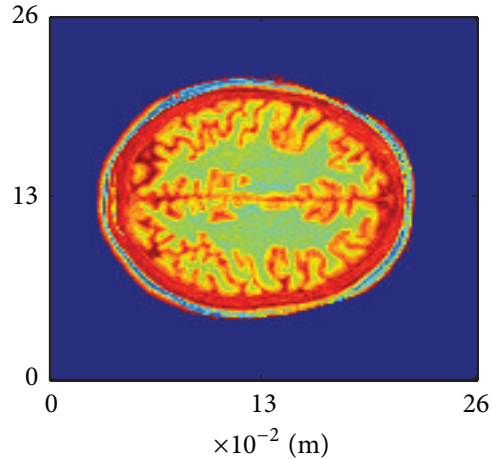

(a)

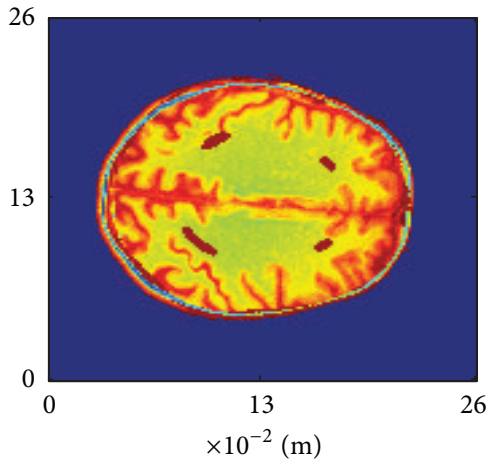

(b)

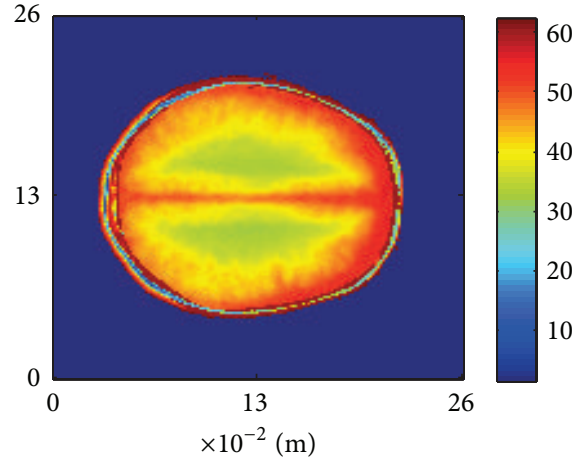

(c)

FIGURE 5: Reconstruction in the absence of stroke. The left panel is the patient's brain map; the middle panel is the result of the reconstruction using the RM initial guess; the right panel shows the reconstruction using the AMM initial guess.

TABLE 1: Time needed for stroke detection for the three cases of initial guesses, in a PC with $2.66 \mathrm{GHz}$ and $3.25 \mathrm{~GB}$ RAM.

\begin{tabular}{lccc}
\hline Method & AMM & RM & ADC-PPS \\
\hline Time (s) & 540 & 660 & 4500 \\
\hline
\end{tabular}

that obtained with the ADC-PPS, which allows the medical personnel to provide an accurate diagnosis of the brain stroke.

\section{Conclusions}

We present a technique for brain stroke detection, whose key feature is the use of an appropriate initial guess for the iterative algorithm of structural inversion. The significant difficulty of this detection by microwaves is due to the barrier of the skull and to the similarity between cerebral fluid dielectric properties and those of blood. We show that the detection problem, however, can be rapidly and successfully solved if the interior of the brain is approximated by either the AMM or the RM initial guesses, which are derived from a $2 \mathrm{D}$ brain map database. In both cases, the detection takes only a few minutes and is much faster than using, as initial guess, the domain map constructed with the mean value of the dielectric parameters. The location of the stroke is exact, even for lesions profoundly situated in the brain, although the size is not well approximated in all the cases. The results confirm that the multifrequency approach using the microwaves in the range of $(0.3-1) \times 10^{9} \mathrm{~Hz}$ and an appropriate initial guess are effective for stroke detection, proving that the microwave imaging has a high potential as a rapid, cost effective, and noninvasive system. Moreover, the quality of the final reconstruction image facilitates to the medical personnel an accurate diagnosis of brain stroke.

\section{Disclosure}

Data used in the preparation of this paper were obtained from the Parkinson's Progression Markers Initiative (PPMI) database (http://www.ppmi-info.org/data). As such, the investigators within PPMI contributed to the design and implementation of PPMI and/or provided data but did not participate in the analysis or writing of this report. PPMI investigators include (complete listing at PPMI site).

\section{Acknowledgments}

The authors would like to thank the Department of Material Science-Engineering and Chemical Engineering for financial support and the Parkinson's Progression Markers Initiative (PPMI) for the access to their database. PPMI-a publicprivate partnership - is funded by the Michael J. Fox Foundation for Parkinson's Research and funding partners, including Abbott, Biogen Idec, F. Hoffmann-La Roche Ltd., GE Healthcare, and Genentech and Pfizer Inc.

\section{References}

[1] H. Ma, J. Ly, and G. A. Donnan, "TIA and stroke: a management guide for GPs," Medicine Today, vol. 7, no. 5, pp. 14-23, 2006.

[2] S. Y. Semenov and D. R. Corfield, "Microwave tomography for brain imaging: feasibility assessment for stroke detection," International Journal of Antennas and Propagation, vol. 2008, Article ID 254830, 8 pages, 2008.

[3] W. T. Joines, Y. Zhang, C. Li, and R. L. Jirtle, "The measured electrical properties of normal and malignant human tissues from 50 to $900 \mathrm{MHz}$," Medical Physics, vol. 21, no. 4, pp. 547550, 1994.

[4] S. Y. Semenov, R. H. Svenson, and G. P. Tatsis, "Microwave spectroscopy of myocardial ischemia and infarction. 1. Experimental study," Annals of Biomedical Engineering, vol. 28, no. 1, pp. 48$54,2000$.

[5] S. Y. Semenov, R. H. Svenson, V. G. Posukh et al., "Dielectrical spectroscopy of canine myocardium during acute ischemia and hypoxia at frequency spectrum from $100 \mathrm{kHz}$ to $6 \mathrm{GHz}$," IEEE Transactions on Medical Imaging, vol. 21, no. 6, pp. 703-707, 2002.

[6] K. W. Muir, A. Buchan, R. von Kummer, J. Rother, and J. C. Baron, "Imaging of acute stroke," Lancet Neurology, vol. 5, no. 9, pp. 755-768, 2006.

[7] D. Ireland and M. Bialkowski, "Feasibility study on microwave stroke detection using a realistic phantom and the FDTD 
method," in Proceedings of the Asia-Pacific Microwave Conference (APMC '10), pp. 1360-1363, December 2010.

[8] D. Ireland and M. Bialkowski, "Microwave head imaging for stroke detection," Progress in Electromagnetic Reseach, vol. 21, pp. 163-175, 2011.

[9] N. Irishina, D. Álvarez, O. Dorn, and M. Moscoso, "Structural level set inversion for microwave breast screening," Inverse Problems, vol. 26, no. 3, Article ID 035015, 2010.

[10] D. Andreucetti, R. Fossi, and C. Petrucci, "Internet resource for the calculation of the dielectric properties of body tissues in frequency range $10 \mathrm{~Hz}-100 \mathrm{GHz}$," 2010, http://niremf.ifac.cnr. it/tissprop/.

[11] Image Data Archive at the Laboratory of Neuro Imaging, https://ida.loni.ucla.edu/.

[12] O. Dorn, E. L. Miller, and C. M. Rappaport, "A shape reconstruction method for electromagnetic tomography using adjoint fields and level sets," Inverse Problems, vol. 16, no. 5, pp. 11191156, 2000.

[13] C. Gabriel, S. Gabriel, and E. Corthout, "The dielectric properties of biological tissues. III: parametric models for the dielectric spectrum of tissue," Physics in Medicine and Biology, vol. 41, no. 11, pp. 2271-2293, 1996. 


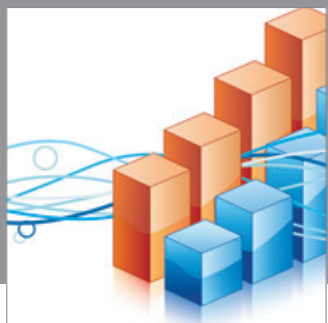

Advances in

Operations Research

mansans

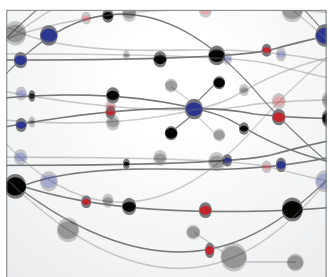

The Scientific World Journal
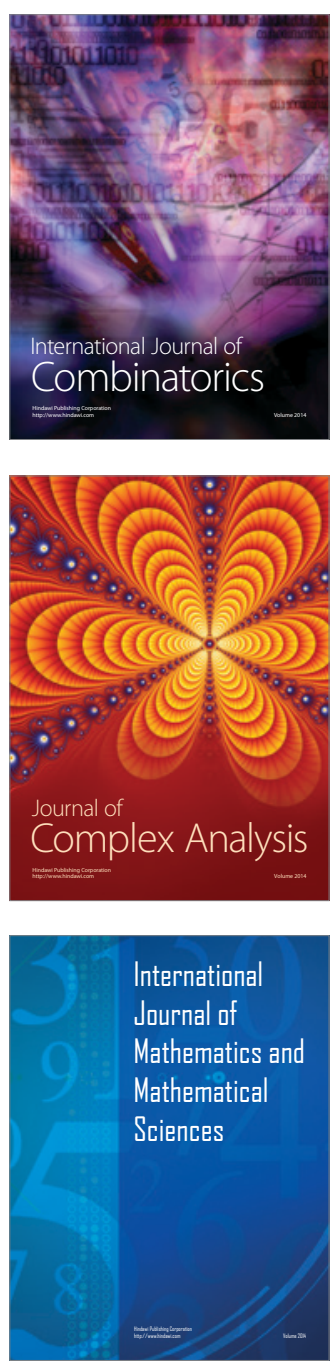
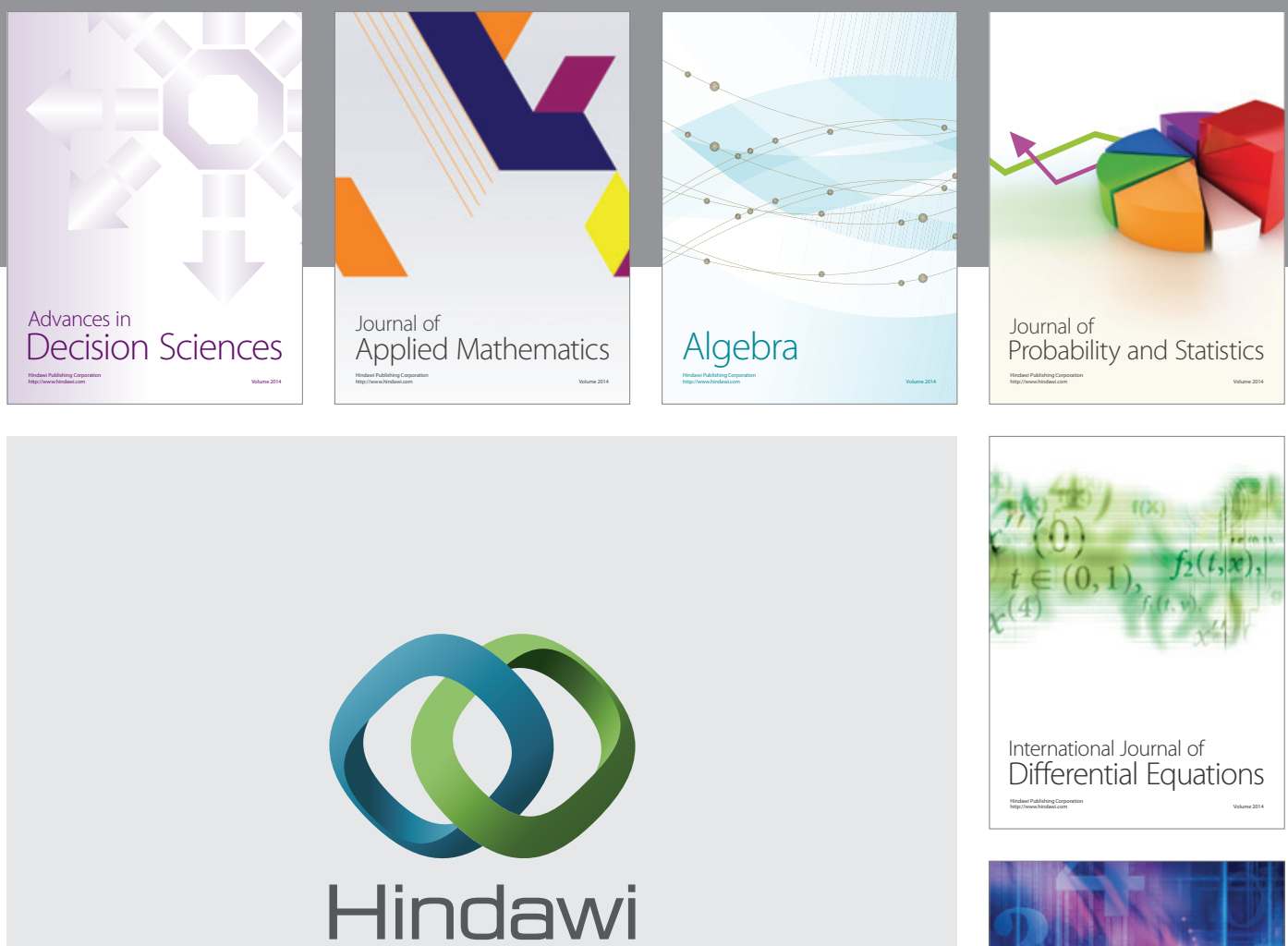

Submit your manuscripts at http://www.hindawi.com
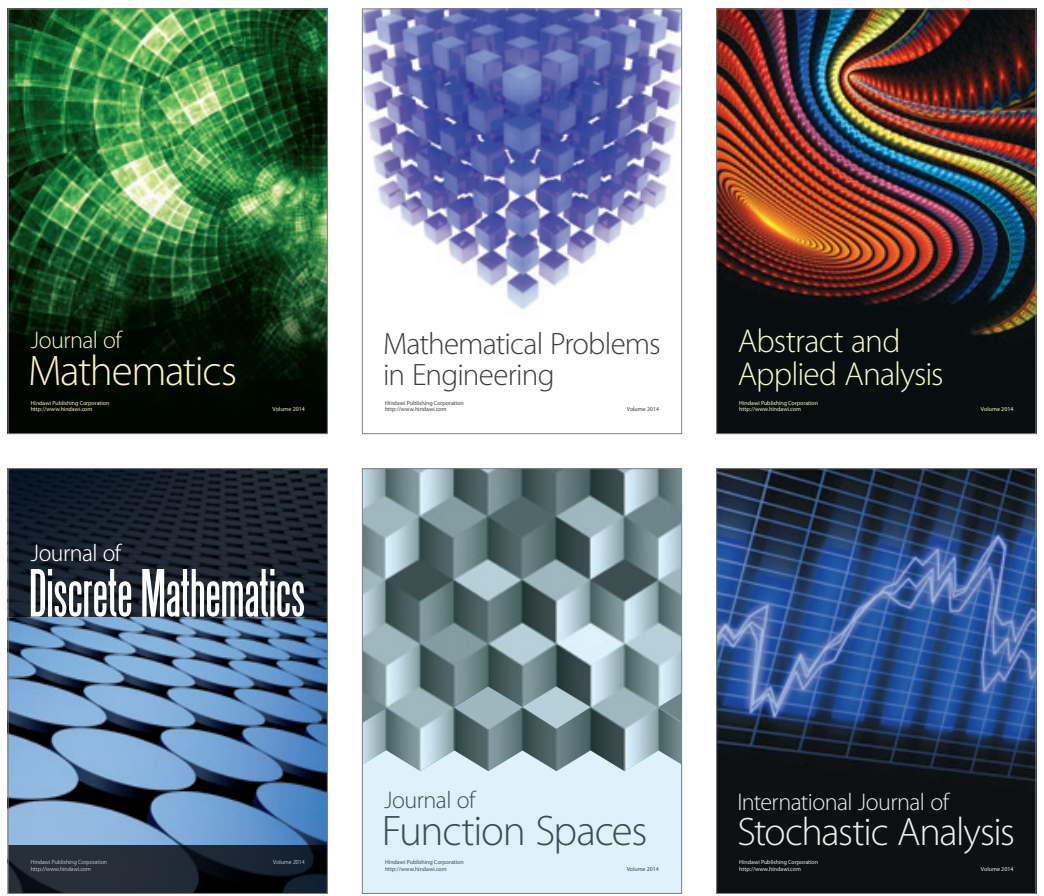

Journal of

Function Spaces

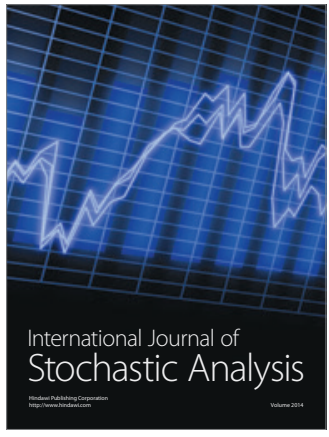

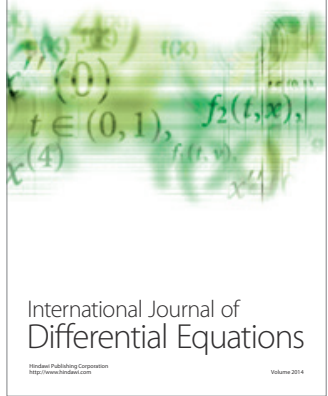
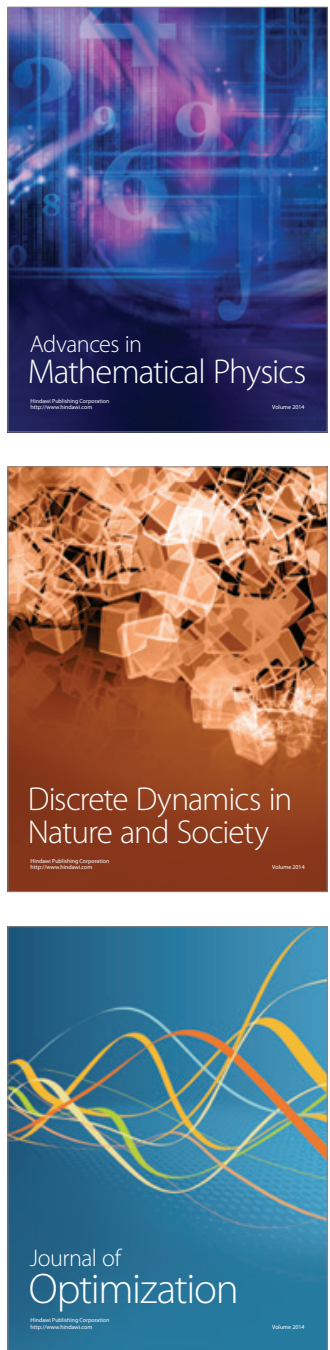